\title{
Role of Machine Learning in WSN and VANETs
}

\author{
Maryam Gillani ${ }^{1}$, Hafiz Adnan Niaz ${ }^{2, *}$, Muhammad Tayyab ${ }^{2}$ \\ ${ }^{1}$ School of Computer Science, University College Dublin, Ireland, \\ Email: maryam.gillani@ucdconnect.ie \\ ${ }^{2}$ College of EME-National University of Sciences and Technology NUST, Islamabad, Pakistan, \\ Email: adnanniaz77@ce.ceme.edu.pk,mtayyab.ee19ceme@ee.edu.pk \\ *Corresponding author, adnanniaz77@ce.ceme.edu.pk
}

\begin{abstract}
Dynamic nature of Vehicular Ad-hoc Networks (VANETs) and Wireless Sensor Networks (WSN) makes them hard to deal accordingly. For such dynamicity, Machine learning (ML) approaches are considered favourable. ML can be described as the process or method of self-learning without human intervention that can assist through various tools to deal with heterogeneous data to attain maximum benefits from the network. In this paper, a quick summary of primary ML concepts are discussed along with several algorithms based on ML for WSN and VANETs. Afterwards, ML based WSN and VANETs application, open issues, challenges of rapidly changing networks and various algorithms in relation to $M L$ models and techniques are discussed. We have listed some of the ML techniques to take additional consideration of this emergent field. A summary is given for ML techniques application with their complexities to cover on open issues to kick start further research investigation. This paper provides excellent coverage of state-of-the-art ML applications that are being used in WSN and VANETs with their comparative analysis.
\end{abstract}

Keywords: Intelligent Systems (IS), Machine Learning (ML), Taxonomy, VANETs, WSN.

\section{INTRODUCTION}

Wireless Sensor Networks (WSN) depends on gathering data and forwarding them to the base stations for further data processing [1]. WSN technologies encounter many hurdles such as insufficient quality of service (QoS), fragile vehicle to infrastructure and connection (V2I), channel access delay, congestion and data packets loss [2]. A considerable amount of work is being done to address the limitations of these challenges, but their complex and rapidly changing nature raises many issues. The 3GPP (3rd Generation Partnership Project) took initiative to examine aiding V2X vehicle toeverything services in the upcoming cellular systems $(5 \mathrm{G})$ and the long term evolution network LTE [3]. Also, graphtheoretic tools along with many effective protocols are designed for resource management in vehicular technologies. Machine learning is anchoring its roots in making 5G, LTE and other related advanced technologies capable of achieving desired and better results effectiveness [4].

Meanwhile, a vast variety of sensors are incorporated into intelligent vehicles for accomplishing various tasks and to help the vehicle collect data from the surrounding environment and monitoring desired operations in real-time. These sensing techniques are making vehicles as intelligent computing systems for data processing and networking capabilities from a modest transportation facility. In VANETs, all nodes are considered as self-sufficient systems that are capable of performing specific operations without any prior data assistance. VANET needs Access Point (AP) for generally associating with the network. Vehicle act as a central point of the vehicular system. VANET works by identifying V2V distance, functional ratio, and V2I access point. Artificial Intelligence (AI) techniques have been introduced in VANETs for speech/image evaluation and data communications among V2V and V2I.

AI is successfully achieving success over various limitations and challenges associated with VANETs. Moreover, data-driven approaches are determined by the success of the pattern recognition techniques related to ML. These tools are working on the backpropagation of the Expectation-Maximization (EM) algorithm, Q-learning, and adaptive learning rate schedules. Machine Learning along with Deep Learning (DL) have catered significant attention in current years due to their outstanding performance in Intelligent Systems (IS) to work in real-time and complex environments with countless successful and effective applications for classification and dealing with robotics.

This paper gives a brief overview of WSN and VANETs with brief key concepts of machine learning. Further, we proceed by providing a taxonomy of ML categories applications in Figure 1. The literature review is given in section II. Section III presents some applications for ML and WSN. In section IV, we have discussed and underline numerous open research issues with limitations associated with ML in VANETS and WSN. Section V is based on ML algorithms and their mapping on various WSN and VANETs techniques and models. Section VI explains the conclusion of the paper.

\section{LITERATURE REVIEW}

ML provides useful tools that are applicable for WSN and VANETs in data-generating, communication and, then data sharing in vehicular networks [5]. It also helps the network to make informed and data-driven decisions. ML approaches are separated into four categories i.e. Supervised learning SL, semi-supervised learning SSL, unsupervised learning $\mathrm{UL}$, and reinforcement learning (RL) [6].

Supervised Learning (SL) is utilized in applications that are supported by enough pre-established datasets along with other information like channel decoder and email spam classification. It can additionally characterize by 
classification and regression. Labels are numerical for regression and categorical for classification. Some other algorithms included are k-nearest neighbours (KNN) [7], Neural Networks [8], Bayesian classifier [9], Support Vector Machine (SVM) [10] and decision trees [11]. on Explaining all the categories and classification precisely. However, only a few of them are considered for discussion in the later sections. This paper comprehensively covers open research challenges and mapping of machine learning algorithms on WSN and VANETs.

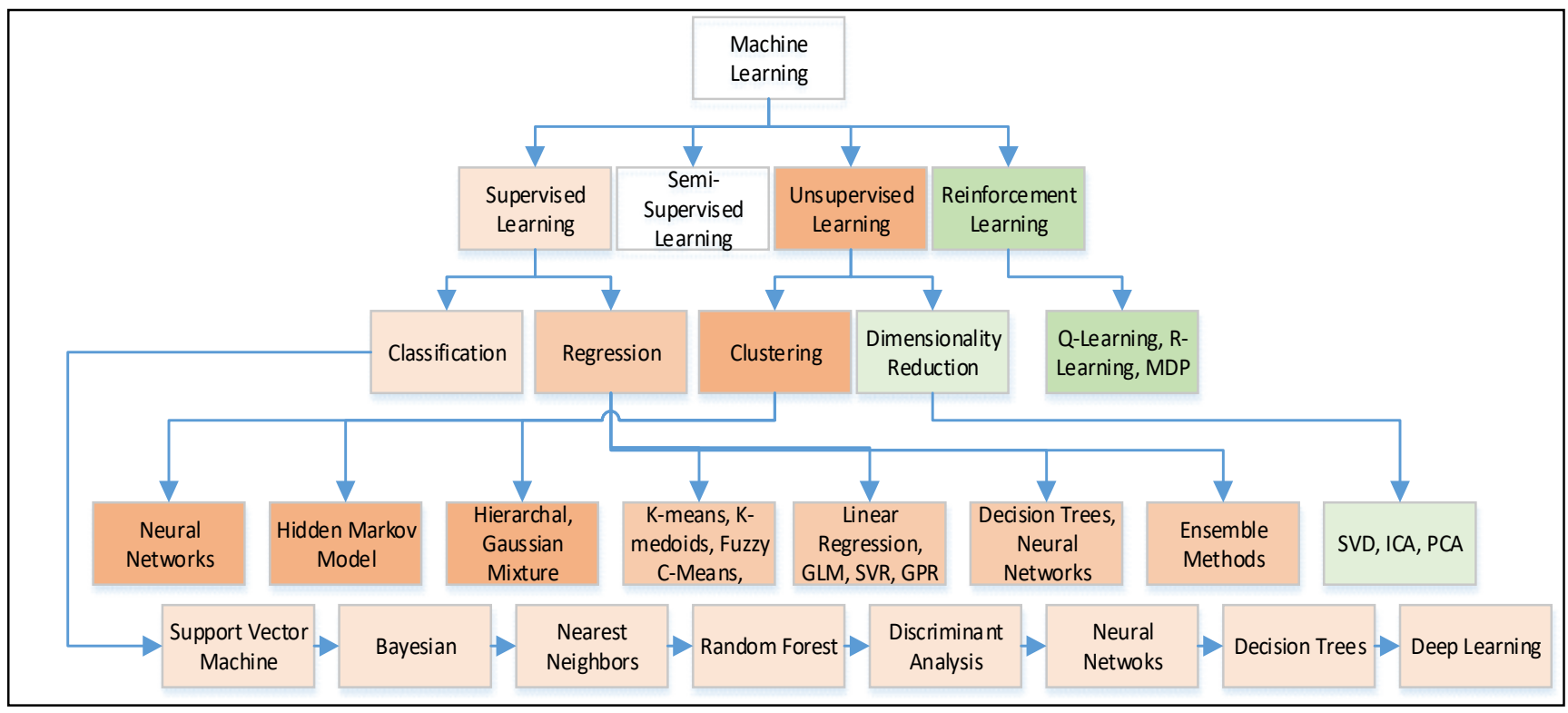

Fig. 1. Taxonomy for machine learning techniques

Unsupervised Learning (UL) deals specifically with unlabelled data with inputs i.e. without any assigned output. In supervised learning, clustering stands for grouping samples. The same clusters grouped with more connections than the samples in other clusters. Standard clustering algorithms are hierarchical clustering, spectrum clustering, and k-means.

Reinforcement learning (RL) is applied to the problems based on sequential decision making in which learner connects with the environment by sequentially taking measures (outputs) on the source of its interpretations (inputs) then getting feedback related to each selected action. These algorithms are applied to robotics, route finding, and gaming. The Semi-Supervised Learning works on both unlabelled and labelled data and it is significant to perform clustering with both. It is favourable for dimensionality reduction for labelled data and regression with unlabelled data. SSL is suitable for real-time applications like natural language processing. Moreover, WSNs are utilizing SSL for the learning process to solve problems like fault detection and localization technique.

Deep learning works by learning data representations from supervised and unsupervised. Reinforcement learning made considerable developments in various ML tasks to incorporate in WSN and VANETs. ML techniques are used in many applications including traffic resource routing, vehicle to vehicle communication, gaming and, speech recognition. Q learning algorithm is used for action space and a larger state. Complex deep reinforcement learning is used with reinforcement learning [10].

In Figure 1, Taxonomy for machine learning techniques is given that explains four major machine learning types with their subtypes, classification and associated techniques and applications. Section V is based

\section{MACHINE LEARNING APPLICATIONS FOR WSN AND VEHICULAR NETWORKS}

Huge Bulk of data produced and accumulated in the vehicular network makes the data-driven methods favourable for decision making that is also applicable to network dynamicity. Machine learning characterizes an effective tool to provide robustness and dynamicity of large data with good performance in WSN and VANETs. Below mentioned are a few of ML applications in WSN and VANETs.

\section{A. Network Safety}

In the intrusion detection system, vehicular networks based on deep neural networks that use unsupervised deep learning to set the parameters and working for the preprocessing stage [11]. In such kinds of techniques, the network can detect and recognize irregularities with a high level of precision. It also forecasts the upcoming word deriving from each vehicle respectively [12].

\section{B. Vehicle Direction Prediction}

Machine Learning is significantly contributing to advanced driver assistance systems (ADAS) for accidental warning and collision avoidance. It also plays a crucial part in networking protocol designs for link scheduling, vehicle routing, and handoff control. Moreover, based on variation GMM and Gaussian mixture models (GMM), probabilistic trajectory prediction has proven to be useful in predicting the vehicle's direction using previously monitored mobility patterns.

\section{Accuracy In-Channel Estimation}

Finding accuracy is a very tedious factor in today's wireless communications systems. Channel estimation is crucial in vehicular networks due to rapidly changing 
topology with short channel interactions and high Doppler shifts [13]. To find accuracy for wireless channels, time and frequency domain correlational time, multipath delay spread, the neighbouring wireless environment, and the vehicle speed location are considered. Data taken from these factors can provide an energetic database to reserve the values of communicating channels. A Variety of ML tools i.e. deep learning and Bayesian learning can be conveniently functional on data prediction, channel accuracy, and gives more trusted channel estimation for present vehicular links [14].

\section{Traffic Flow Prediction}

VANETs hold rapidly changing traffic states and conditions [12]. ML work for the real-time traffic data and traffic information stored by numerous vehicular sensors. Machine learning is effective in enhancing the flow in predicting traffic performance and achieve a real-time response. In [15], a method has been proposed on deep learning for traffic flow prediction. In this method rapidly changing traffic flow to learn generic features and stacked auto encoder is designed. Further, training is performed with a greedy layer-wise pattern.

\section{E. WSN Load Balancing}

Due to the periodicity of data coming from WSN, different patterns and transitions occur to handle by the network. In this scenario, learning-based approaches for balancing the load in WSN can be a handsome choice. The user organization problem with balancing the load in the active environment is already established online [16].

\section{F. Location and Scheduling Routing}

ML assured that predicted dynamics is a feasible option for the networking protocol designs. Hidden Markov model (HMM) is effective in predicting the vehicle's upcoming locations based on past mobility data and mobility in VANET with V2I and V2V links [17].

\section{G. Congestion Control}

Congestion is a significant problem in WSN and VANETs. This issue increases with the network density e.g. more congestion occurs in busy and crowded urban environments. When a huge quantity of vehicles tries for accessing the communication channels altogether, it causes immense data collision with increased data packet loss and bottleneck conditions. ML is effective in data congestion control designing by using k-means clustering [18].

\section{H. Intelligent Wireless Management}

The challenge arises due to the heavy signalling overhead as well as difficulty in to formulate satisfactory heterogeneous wireless and vehicular links. For such challenges, reinforcement learning provides an effective solution to maximize a successful signalling ratio by interacting with the dynamic environment [19].

\section{Routing Techniques}

The Challenge arises due to varying techniques needed for different routing purposes. VANETs are subjected to random and rapidly changing transitions. To establish a design or model, applicable to all kinds of environments is still a difficult task to attain. Despite massive work performed to meet different environmental criteria for WSN and VANETS, a standardized approach is still missing to cater routing needs.

\section{Open RESEARCH CHALLENGES}

Machine Learning has made considerable progress in WSN and VANETs due to its distinguishing nature. It is now sufficient to apply the existing ML algorithms in vehicular networks. Although, finding a suitable ML method for a problem is much challenging. Some of the limitations and open issues are discussed below.

\section{A. Localization}

Existing localization algorithms majorly cover twodimensional space but real-time applications require threedimensional space. Towards three-dimensional spaces for mobile/static VANETs and WSN, it is essential to improve localization techniques [6].

\section{B. Security Problems}

Machine learning is beneficial in dealing with cyberattacks which also affects the security of VANETs. Since, the ML-based scheme can produce unexpected results which also causes security risks [11]. Resultantly even though in WSN areas, ML has attained outstanding enhancements but to improve the security and robustness of ML-based algorithms significant improvements are needed.

\section{Method Complexity}

Conventional ML procedures and techniques require suitable work for feature design. On the other hand, deep neural networks provide optimal achievement through acquiring the features straight from raw data. Therefore, in comparison with traditional methods information may act more effectively in deep neural networks. As a result, few models should be developed and designed to ease the computation resource limitation.

\section{Coverage and Connectivity}

Mostly, in WSN applications, the sensor nodes deployment is unpredictable which causes a coverage hole in the network. It is very difficult to find the coverage hole respectively [6].

\section{E. Anomaly Detection}

The technique of anomaly detection is applicationspecific. Many ML-based techniques have been developed to detect anomalies which causes a tough job to select its algorithm for heterogeneous wireless sensor networks. So the selection of anomaly detection algorithms to heterogeneous WSNs is a tough job. Further research is required about actions needed to be taken for anomaly detection [20].

\section{F. Congestion Avoidance}

To minimize the transmission delays between the nodes, a simple congestion control framework is required at each node because of the limited energy with the limitations of memory in VANETs and WSNs [16]. There is a need to 
improve traffic assessment protocols for classifying rapid and dynamically changing route conditions to bypass congestion in VANETs.

\section{G. $Q O S$}

In WSN, QoS aims to fulfil the requirements for users and applications. Its standards vary from different application nature and requirements [21]. Thus it is a difficult task to define the QoS standards according to different requirements.

\section{H. High Energy and Computing Needs}

Machine Learning based applications require high energy of computing to execute complex and extensive models and methodologies. In WSN and VANETs, high mobility nodes and traffic require extensive energy demands for executing and getting desired results. WSN and VANETs need lightweight and energy-efficient models in relation with ML to meet high energy demand needs.

\section{ML ALGORITHMS MAPPING ON VARIOUS WSN AND VANETS TECHNIQUES AND MODELS}

\section{A. Supervised Learning}

In Supervised learning, various techniques of Machine Learning i.e. Support Vector Machine (SVM), Naive Bayes and K-Nearest Neighbours (KNN) assist in many scenarios to analyse WSN and VANETs systems. Machine learning based strategies are providing tons of features to gain safety, reliability and efficiency for WSN and VANETs.

\section{1) Support Vector Machine (SVM)}

SVM is based on statistical learning theory. It is one of the effective machine learning algorithms for classification purpose with an ability to minimize the empirical error. SVM reduced the structural risks of over-learning with limited samples. For example, there is SVM based localization technique Fast- (SVM) [22] and Naive convex hull algorithm [23] for connectivity and coverage technique. There is another SVMs classification method [13] for fault detection and Efficient SV based clustering protocol [24] for secure routing techniques. SVM is best suited for malicious behaviour detection in VANET system [25].

\section{2) Bayesian}

Based on simple probability theory, Bayes theorem is effective in Predicting traffic crash, possible collisions and warning estimation. For example, Three-level hierarchical prior Model [26] is a reliable localization technique and NBC Naive Bayes classifier [27] is efficient Connectivity and Coverage Technique widely used. Moreover, the Bayesian network model based sensor fault detection scheme [28] and Naïve Base Cluster Head Node [29] are fault detection and Routing Technique.

\section{3) K-Nearest Neighbours (KNN)}

$\mathrm{KNN}$ are effective for classification and regression predictive problems. Their interpretation is easy and usually takes less calculation time. $\mathrm{KNN}$ is used in Intrusion detection system based on K-NN [30]. They are of great importance anomaly detection e.g. KNN-based AD scheme [31] and data aggregation schemes e.g. Bovel (NN) imputation method [32].

\section{4) Random Forest}

A huge part of Machine Learning belongs to Classification. The ability to classify observation and predictions plays a significant role in various VANETS and WSN applications. Random Forest classifier is of prime importance due to its ability to analyse clusters (crowds) intelligently. The situation with massive traffics jams and high traffic roads are better to deal with random forest.

\section{5) Discriminant Analysis}

Discriminant analysis is effective in dimensionality reduction and classification as well. Detecting intrusion in vehicular communication for autonomous cars is best suited with discriminant analysis. It offers great role in secure communication due to their effective ability to diagnose malicious vehicles with ease and less execution time. One such example is the Intelligent Intrusion Detection Scheme that uses Discriminant Analysis for detecting Denial of Service (DoS) and black hole attacks in VANETs [33].

\section{6) Neural Networks}

Neural Networks enables security provision among interacting vehicles that mainly relies on radial basis neural networks and makes it possible in the identification of malicious nodes by indicating abnormal behaviour [34]. Neural Networks are also playing a vital role in helping the driver to control vehicle intelligently along with accidents and obstacles detection.

\section{7) Decision Trees}

Decision Trees are effective in identifying road structure, traffic conditions and flows and help in minimizing time delays, congestion and alerting warning for possible collision incidents. The fuzzy detection tree model is used for traffic signal optimization that effectively deals with extreme traffic and network scenarios [35].

\section{8) Deep Learning}

Deep learning is doing wonders when it comes to intrusion Detection Systems both in WSN and VANETs. To suppress the inter-vehicle attacks and malicious nodes detection, deep learning based distributed approach is widely adapted for abnormal activity detections [36]. Another fault detection technique is Distributed data mining method based on DNN [2] and routing technique is IP Base node degree of WC [37].

\section{B. Unsupervised Learning}

Unsupervised learning in WSN and VANETs are proposing an algorithm that can classify networks into groups (clusters). Unsupervised clustering is more appropriate because of its ability to directly compare different cluster formation. This factor adds reliability and better maintenance for VANETs as well as WSN [38].

\section{1) Hidden Markov Model}

Hidden Markov Model assist in marinating complex endto-end vehicular and network connections in sudden and 
frequent changing nodes/vehicles. One such example is Hidden Markov Model named PRHMM (Predictive Routing based on Hidden Markov Model) which particularly focuses on the vehicle to vehicle and vehicle to infrastructure communications by vehicle density and moving speed [39].

\section{2) Hierarchal \& Gaussian Mixture}

Hierarchal and Gaussian mixture are important due to their tractable analytical properties. It enables smooth communication through a distributed context. They are contributing to direct and indirect Intelligent Transport systems based applications for smart cities. One such example is cooperative positioning with GPS aid in WSN and VANETs [40].

\section{3) SVD, ICA, PCA}

Singular Value decomposition (SVD), Independent Component Analysis (ICA) and Principal Component Analysis (PCA) are machine learning methods that are acting effectively in VANETs and WSN.

\section{Reinforcement Learning}

Reinforcement Learning plays a critical role in the selection of authentication nodes and parameters to save application learning time and improve performance.

\section{1) Q-learning}

Q-learning is functional in traffic aware routing models. Q-learning has experimented high dynamicity and complex network structures. Q-learning-based TrafficAware Routing (QTAR) utilizes dynamic selection for packets being exchanged among different nodes and vehicles. Q-learning has resolved the unicast problem among WSN and VANETs successfully through targeted neighbour intersections [41].

\section{2) Markov Decision Process}

Markov Decision Process (MDP) helps in making simple assumptions to resolve complex networks connectivity issues by using Q-learning. MDP-based transmission policies Rate and Power (MDPRP) uses MDP to prevent network congestion through maximizing channel utilization and enabling secure performance for safety applications [42].

\section{CONCLUSION AND FUTURE WORK}

Concluding all, we have presented the basics of machine learning briefly and summarized the ML-based challenges, limitations, and open issues for WSNs and VANETs in this paper. We have also discussed the new advances of ML to track the dynamics of vehicular environments and WSN. Various ML techniques are mapped based on their complexity upon some open issues that require considerable attention. Although, research in this domain is still in its way to progress but we have tried to map existing knowledge to explore new horizons of possibilities. Many of the research gap's problems are yet to be explored and shall be completely defined. Through above mentioned discussion and examples, we can say that Machine Learning is effectively providing the solution to many technical problems related to WSN and VANETs that were once considered to be difficult to accomplish.

\section{REFERENCES}

[1] L. Liang, H. Peng, G. Ye Li and X. Shen, "Vehicular Communications: A Physical Layer Perspective," IEEE Transactions on Vehicular Technology, vol.66, no.12, pp. 10647 - 10659, 2017.

[2] L. Chunlin, X. Xiaofu and H. Yuejiang, "Distributed Data Mining Based on Deep Neural Network for Wireless Sensor Network," International Journal of Distributed Sensor Networks, vol.11, no.7, pp. 1-7, 2015.

[3] Y.L. Tseng, "LTE-Advanced Enhancement for Vehicular Communication," IEEE Wireless Communications, vol.22, no.6, pp. 4-7, 2015.

[4] L. Liang, S. Xie, G. Ye Li, Z. Ding and X. Yu, "Graph-Based Resource Sharing in Vehicular Communication," IEEE Transactions on Wireless Communications, vol.17, no.8, pp. 4579-4592, 2018.

[5] C. Jiang, H. Zhang, Y. Ren, Z. Han, K.C. Chen and L. Hanzo, "Machine Learning Paradigms for Next-Generation Wireless Networks," IEEE Wireless Communications, vol.24, no.2, pp.98-105, 2017.

[6] H. Ye, L. Liang, G.Y. Li, J. Kim, L. Lu and M. Wu, "Machine Learning for Vehicular Networks," IEEE Vehicular Technology Magazine, vol.2, pp. 1-16, 2018.

[7] K. Beyer, J. Goldstein, R. Ramakrishnan and U. Shaft, "When Is "Nearest Neighbor" Meaningful?" 1999 International Conference Jerusalem(ICDT), pp. 217-235, 1999.

[8] Y. LeCun, Y. Bengio and G. Hinton, "Deep Learning," Nature, vol.521, no.7553, pp. 436-444, 2015.

[9] G.E. Box and T.G.C., Bayesian inference in statistical analysis, John Wiley \& Sons, 2011, Canada.

[10] N.C. Luong, D.T. Hoang, S. Gong, D. Niyato, P. Wang and Y. Chang, "Applications of Deep Reinforcement Learning in Communications and Networking: A Survey," IEEE Communications Surveys \& Tutorials, vol.21, no.4, pp. 3133 - 3174, 2019.

[11] D. Amodei, C. Olah, J. Steinhardt, P. Christiano, J. Schulman and D. Mané, "Concrete problems in AI safety," arXiv preprint arXiv:1606.06565, 2016.

[12] M. Gillani, A. Ullah and H. A. Niaz, "Trust Management Schemes for Secure Routing in VANETs - A Survey," 2018 International Conference on Mathematics, Actuarial Science, Computer Science and Statistics (MACS), pp.1-6, 2018. DOI: 10.1109/MACS.2018.8628440

[13] S. Zidi, T. Moulahi and B. Alaya, "Fault Detection in Wireless Sensor Networks Through SVM Classifier," IEEE Sensors Journal, vol.18, no.1, pp.340-347, 2018

[14] R.F. Atallah, C.M. Assi and J.Y. Yu, "A Reinforcement Learning Technique for Optimizing Downlink Scheduling in an EnergyLimited Vehicular Networ," IEEE Transactions on Vehicular Technology, vol.66, no.6, pp.4592-4601, 2017.

[15] Y. Lv, Y. Duan, W. Kang, Z. Li and F.Y. Wang, "Traffic Flow Prediction with Big Data: A Deep Learning Approach," IEEE Transactions on Intelligent Transportation Systems, vol.16, no.2, pp.865-873, 2015.

[16] Z. Li, C. Wang and C.J. Jiang, "User Association for Load Balancing in Vehicular Networks: An Online Reinforcement Learning Approach," IEEE Transactions on Intelligent Transportation Systems, vol.18, no.8, pp.2217-2228, 2018.

[17] L. Yao, J. Wang, X. Wang, A. Chen and Y. Wang, "V2X Routing in a VANET Based on the Hidden Markov Model," IEEE Transactions on Intelligent Transportation Systems, vol.9, no.13, pp.889-899, 2017.

[18] N. Taherkhani and S. Pierre, "Centralized and Localized Data Congestion Control Strategy for Vehicular Ad Hoc Networks Using a Machine Learning Clustering Algorithm," IEEE Transactions on Intelligent Transportation Systems, vol.17, no.11, pp.3275-3285, 2016.

[19] Y. He, N. Zhao and H. Yin, "Integrated Networking, Caching, and Computing for Connected Vehicles: A Deep Reinforcement Learning Approach," IEEE Transactions on Vehicular Technology, vol.67, no.1, pp.44-55, 2018.

[20] H.S. Emadi and S.M. Mazinani, "A Novel Anomaly Detection Algorithm Using DBSCAN and SVM in Wireless Sensor Networks," Wireless Personal Communications, vol.98, pp.2025-2035, 2018.

[21] M. Gillani, A. Ullah and H.A. Niaz, "Survey of Requirement Management Techniques for Safety Critical Systems," 2018 
International Conference on Mathematics, Actuarial Science, Computer Science and Statistics (MACS), pp.1-5, 2018.

[22] F. Zhu and J. Wei, "Localization Algorithm for Large Scale Wireless Sensor Networks Based on Fast-SVM," Wireless Personal Communications, vol. 95, no. 3, p. 1859-1875, 2017.

[23] W. Kim, M.S. Stanković, K.H. Johansson and H.J. Kim, "A Distributed Support Vector Machine Learning Over Wireless Sensor Networks," IEEE Transactions on Cybernetics, vol.45, no.11, pp.2599-2611, 2015.

[24] F. Khan, S. Memon and S.H. Jokhio, "Support Vector Machine Based Energy Aware Routing in Wireless Sensor Networks," 2016 International Conference on Robotics and Artificial Intelligence (ICRAI), pp.1-4, 2016. DOI: 10.1109/ICRAI.2016.7791218

[25] S. Khatri, H. Vachhani, S. Shah, J. Bhatia, M. Chaturvedi and S. Tanwar, "Machine Learning Models and Techniques for Vanet Based Traffic Management: Implementation Issues and Challenges," Peerto-Peer Networking and Applications, no. 14, pp. 1778-1805, 2021.

[26] Y. Guo, B. Sun, N. Li and D. Fang, "Variational Bayesian InferenceBased Counting and Localization for Off-Grid Targets with Faulty Prior Information in Wireless Sensor Networks," IEEE Transactions on Communications, vol.66, no.3, pp.1273-1283, 2018.

[27] B. Yang, Y. Lei and B. Yan, "Distributed Multi-Human Location Algorithm Using Naive Bayes Classifier for a Binary Pyroelectric Infrared Sensor Tracking System," IEEE Sensors Journal, vol.16, no. 1, pp.216-223, 2016.

[28] H. Zhang, J. Liu and N. Kato, "Threshold Tuning-Based Wearable Sensor Fault Detection for Reliable Medical Monitoring Using Bayesian Network Model," IEEE Systems Journal, vol.12, no.2, pp.1886-1898, 2018

[29] V. Jafarizadeh, A. Keshavarzi and T. Derikvand, "Efficient Cluster Head Selection Using Naïve Bayes Classifier for Wireless Sensor Networks," Wireless Networks, vol.23, p.779-785, 2017.

[30] W. Li, P. Yi, Y. Wu, L. Pan and J. Li, "A New Intrusion Detection System Based on KNN Classification Algorithm in Wireless Sensor Network," Journal of Electrical and Computer Engineering, vol. 2014, pp. 1-8, 2014.

[31] M. Xie, J. Hu, S.Han and H.H. Chen, "Scalable Hypergrid k-NNBased Online Anomaly Detection in Wireless Sensor Networks," IEEE Transactions on Parallel and Distributed Systems, vol.24, no.8, pp.1661-1670, 2013

[32] Y. Li and L.E. Parkerb, "Nearest Neighbor Imputation Using SpatialTemporal Correlations in Wireless Sensor Networks," Information Fusion, vol.15, pp.64-79, 2014

[33] K.M.A. Alheeti, A. Gruebler and K.M. D. Maier, "Using Discriminant Analysis to Detect Intrusions in External Communication for Self-Driving Vehicles," Digital Communications and Networks, Science Direct, vol.3, no.3, pp.180-187, 2017.

[34] T. Ovasapyan, D. Moskvin and M. Kalinin, "Using Neural Networks to Detect Internal Intruders in VANETs," Automatic Control and Computer Sciences, vol. 52, no.8, pp. 954-958, 2018.

[35] M. Balta and İ. Özçelik, "A 3-Stage Fuzzy-Decision Tree Model for Traffic Signal Optimization in Urban City Via A SDN Based VANET Architecture," Future Generation Computer Systems, vol. 104, pp. 142-158, 2020.

[36] J. Shu, L. Zhou, W. Zhang, X. Du and M. Guizani, "Collaborative Intrusion Detection for VANETs: A Deep Learning-Based Distributed SDN Approach," IEEE Transactions on Intelligent Transportation Systems, pp. 1-12, 2020. DOI: 10.1109/TITS.2020.3027390

[37] Y. MinLee, "Classification of Node Degree Based on Deep Learning and Routing Method Applied for Virtual Route Assignment," Ad Hoc Networks, vol. 58, pp. 70-85, 2017.

[38] Z. Khan, P. Fan, S. Fang and F. Abbas, "An Unsupervised ClusterBased VANET-Oriented Evolving Graph (CVoEG) Model and Associated Reliable Routing Scheme," IEEE Transactions on Intelligent Transportation Systems, vol.20, no.10, pp.3844-3859, 2019.

[39] Y. Lin, W. Jie, W. Xin, C. Ailun and W. Yuqi, "V2X Routing in a VANET Based on the Hidden Markov Model," IEEE Transactions on Intelligent Transportation Systems, vol.19, no.3, pp.889-899, 2018.

[40] H. Gia-Minh, D. Benoit, H. Jerome and S. Dirk, "On Communication Aspects of Particle-Based Cooperative Positioning in GPS-aided VANETs," 2016 IEEE Intelligent Vehicles Symposium (IV), pp.20$25,2016$.

[41] J. Wu, M. Fang, H. Li and X. Li, "RSU-Assisted Traffic-Aware Routing Based on Reinforcement Learning for Urban Vanets," IEEE Access, vol.8, pp.5733-5748, 2020.
[42] J. Aznar-Poveda, A.J.G. Sanchez, E.E. Lopez and J.G. Haro, "MDPRP: A Q-Learning Approach for the Joint Control of Beaconing Rate and Transmission Power in VANETs," IEEE Access, vol.9, pp.10166-10178, 2021. 